\title{
PHASE TRANSITIONS AND STABILITY OF GROUP IV BCC METALS
}

\author{
M.L. Verma, A. Verma \\ Department of Physics, G.G.D.S.D. College, Palwal-121102, India \\ AND R.P.S. RATHORE \\ Department of Physics, R.B.S. College, Agra-282002, India
}

(Received April 9, 1997; revised version August 22, 1997; in final form December 18, 1997)

Born stability criteria were employed in the framework of extended generalised exponential potential to calculate the theoretical strength and range of stability of a perfect uniaxially stressed crystal lattice of group IV bcc metals: titanium, zirconium, and hafnium. The analysis depicts two ranges of stability, a bcc phase and a body centered tetragonal phase. The computed value of theoretical strength and strain of bcc Ti, $\mathrm{Zr}$ and Hf agree satisfactorily with the experiments limits. The second-order elastic constants $C_{11}$ and $C_{12}$ were also computed.

PACS numbers: 62.20 .Dc

\section{Introduction}

The phase transitions in high-temperature superconductors (HTS) as well as in metals of bcc and fcc configuration have assumed interesting and significant dimensions in the theoretical and experimental fields. It has been established by Abrikosov et al. [1] that the twinning in high-temperature superconductors is due to the phase transition from the tetragonal to orthorhombic structure and the twinning planes usually form periodic structure with the distance [2] between twinning planes in the range 200-2000 $\AA$. Also, there are many strong evidences [3-12] that the elastic and lattice dynamical properties of high-temperature superconductors are considerably affected by phase transitions from tetragonal to orthorhombic structure: Encouraged by these studies, we have been motivated to undertake the study of elastic behaviour during phase transitions initially from cubic to tetragonal structure and the stability of the metals of bcc origin. The study can later be extended for phase transitions from tetragonal to orthorhombic structure. 
Many authors [13] have estimated the theoretical or ideal strength of perfect crystals using a variety of theoretical models. In fact; ideal strength means the theoretical upper limit to the maximum applied stress of a particular nature on a perfect specimen of the material before it becomes mechanically unstable [14]. Since a perfect crystal lattice is homogeneous throughout, it cannot become mechanically unstable till the system lowers its total energy by spontaneously undergoing uniform deformations, usually called twinning or nucleation $[1,15]$.

Taylor [16] and Wassermann [17] have shown that the strength of crystals may be increased many times when their size is made sufficiently small. Further, the problem of determining the ideal strength of a perfect crystal is of fundamental importance because it is thought that the strength of some metallic whiskers or fine filaments approach the theoretical limit. In addition, the problem of calculating the theoretical strength of perfect crystals is relevant to our understanding of many phenomena occurring in solid state physics such as deformation of whiskers, martensitic transformation, efficiency of grinding processes and mechanical properties of small structures such as metallized integrated circuit structures.

Because of numerous applications of the problem of theoretical strength of solids and widespread interest in whiskers and their impact on modern theory of solids, many workers [18] have studied this problem of theoretical strength both for undeformed and deformed crystal lattices with various modes of deformations and with various forms of interactions between the atoms. Recently, Singh [19] has computed theoretical strength of noble metals in [100] direction by using a pseudopotential approach and has made a rigorous estimation of binding energy. Needless to say our present knowledge of interatomic interactions in solids is still handicapped by many constraints and therefore an accurate quantitative calculation of the theoretical strength for most of the solids is hardly tractable. Even though, by using pseudopotential models or more simplified model potentials, it has been attempted to gain semiquantitative information about the theoretical strength and range of stability for some crystalline solids $[19,20]$.

The purpose of the present communication is to apply Porn stability criteria [1] using the extended generalised exponential potential (EGEP) to the problem involving any kind of lattice deformation, that is, either homogeneous expansion or contraction and interpret the results of detailed numerical computations carried out for bcc titanium (Ti), zirconium ( $\mathrm{Zr}$ ) and hafnium (Hf). The theoretical strength and range of stability of bcc metals namely $\mathrm{V}, \mathrm{Nb}$ and $\mathrm{Ta}$ of group VA of periodic table have recently been computed [20] successfully in the framework of present potential. The presently computed values of theoretical strength and strain for bcc $\mathrm{Ti}, \mathrm{Zr}$ and $\mathrm{Hf}$ agree reasonably well with the experimental limits.

\section{Theory}

\subsection{Extended generalised exponential potential}

The extended generalised exponential potential representing the true and realistic nature of the repulsive as well as the attractive components of the interactions coupling the $i$-th and $j$-th atoms separated by a distance $r_{i j}$ assumes the form

$$
\Phi_{m}\left(r_{i j}\right)=[D /(m-1)]\left[\mathrm{e}^{-m \alpha\left(r_{i j}-r_{0}\right)} /\left(\alpha r_{i j}\right)^{n}-m\left(\alpha r_{i j}\right)^{n} \mathrm{e}^{-\alpha\left(r_{i j}-r_{0}\right)}\right],
$$


where $m$ and $n$ are the parameters which take care of electronic exchange and correlation effects and the three-body forces such as volume forces in an alternative and simpler form, respectively. $D$ and $\alpha$ are parameters with dimensions of energy and (distance $)^{-1}$ respectively, such that $D$ is depth parameter and stands for dissociation energy of the pair and $\alpha$, the width parameter, is a measure of the hardness of the potential. Finally, $r_{0}$ stands for the equilibrium separation parameter.

The details concerning the evaluation of the parameters of the potential are discussed in our earlier paper [21]. The input data for bec metals studied and their computed potential parameters are given in Table I and Table II, respectively.

TABLE I

Input data for bcc metals.

\begin{tabular}{c|c|c}
\hline \hline Metal & $\begin{array}{c}\text { Semi-lattice } \\
\text { constant } \\
{\left[\times 10^{-10} \mathrm{~m}\right]}\end{array}$ & $\begin{array}{c}\text { Bulk } \\
\text { modulus } \\
{\left[\times 10^{11} \mathrm{~N} / \mathrm{m}^{2}\right]}\end{array}$ \\
\hline $\mathrm{Ti}$ & 1.664 & 1.180 \\
$\mathrm{Zr}$ & 1.812 & 0.870 \\
$\mathrm{Hf}$ & 1.805 & 1.123
\end{tabular}

TABLE II

Computed potential parameters for bcc metals.

\begin{tabular}{c|c|c|c|c|r|c|c}
\hline \hline Met. & $n$ & $m$ & $\alpha a_{0}$ & $\alpha \times 10^{10} \mathrm{~m}^{-1}$ & \multicolumn{1}{|c|}{$\beta$} & $D \times 10^{-21} \mathrm{~J}$ & $r_{0} \times 10^{-10} \mathrm{~m}$ \\
\hline $\mathrm{Ti}$ & 1 & 2.0 & 1.87350 & 1.125901 & 465.7123 & 1.257433 & 5.456577 \\
$\mathrm{Zr}$ & 1 & 1.5 & 1.78080 & 0.982781 & 4878.8660 & 0.110512 & 8.641461 \\
$\mathrm{Hf}$ & 2 & 1.5 & 2.04445 & 1.132659 & 1761365.0000 & 0.000083 & 12.697200
\end{tabular}

\subsection{Theoretical considerations}

The necessary and sufficient conditions for a lattice to be in stable equilibrium, as has been discussed in considerable detail by Milstein [18], under the effect of uniaxial stress in [100] direction in terms of Born stability criteria take the form

$$
\begin{aligned}
& B_{11}>0, \quad B_{22}>0, \quad B_{12}>0, \quad B_{23}>0, \\
& A A=B_{22}-B_{23}>0, \\
& B B=B_{11}\left(B_{22}+B_{23}\right)-2\left(B_{12}\right)^{2}>0 .
\end{aligned}
$$

The moduli $B_{i j}$, internal energy $(E)$ per unit cell and the stress acting normal to the face of the unit cell in the direction $a_{1}$ are represented by the following equations:

$$
B_{i j}=\left[\frac{\partial^{2} E}{\partial a_{i} \partial a_{j}}\right],
$$




$$
\begin{aligned}
& E=(1 / 2) n^{\prime} \sum_{l_{1} l_{2} l_{3}} \phi(r), \\
& \sigma=\frac{1}{a_{2} a_{3}}\left[\frac{\partial E}{\partial a_{1}}\right],
\end{aligned}
$$

where $i$ and $j$ have their values ranging from 1 to 6 and $n^{\prime}$ is the number of atoms per unit cell. Also $a_{1}, a_{2}$ and $a_{3}$ refer to semi-lattice constants and $a_{4}, a_{5}$ and $a_{6}$ are the angles between $a_{2}$ and $a_{3}, a_{3}$ and $a_{1}, a_{1}$ and $a_{2}$, respectively, and for a tetragonal crystal lattice

$$
\begin{aligned}
& a_{2}=a_{3} \text { and } a_{4}=a_{5}=a_{6}=\pi / 2, \\
& r=\left[l_{1}^{2} a_{1}^{2}+\left(l_{2}^{2}+l_{3}^{2}\right) a_{2}^{2}\right]^{1 / 2} .
\end{aligned}
$$

The other details concerning the formulation of theoretical strength and its computation are given in our recent paper [20].

\section{Results and discussion}

A close review of the literature reveals that a variety of metallic whiskers have exhibited maximum stresses in the range of about $0.17 \times 10^{10} \mathrm{~N} / \mathrm{m}^{2}$ (for $\mathrm{Ag}$ ) to about $1.31 \times 10^{10} \mathrm{~N} / \mathrm{m}^{2}$ (for $\mathrm{Fe}$ ) with a corresponding strains estimated to be about $3 \%$ to $5 \%$ [22-24].

The instability in compression and in tension for the bcc lattice results from the violation of the conditions defined by Eq. (3) and Eq. (4), respectively.

The body centred tetragonal (bct) lattice fails in compression by violating the condition defined by Eq. (4) as a result of its inability to support an additional compressive load, while in tension by violating the condition $B_{23}>0$ wherein the angle $a_{4}$ (the angle between $a_{2}$ and $a_{3}$ ) deviates from $90^{\circ}$.

We now discuss below our findings for bcc metallic crystals in the framework of extended generalised exponential potential, concerning the moduli $B_{i j}$, applied stress and internal energy.

\subsection{Titanium}

Figure 1 shows the variation of $A A=B_{22}-B_{23}$ and $B B=B_{11}\left(B_{22}+B_{23}\right)$ $-2 B_{12}^{2}$ as a function of semi-lattice constant $a_{1}$ for Ti. Figure 2 shows the variation of applied stress and internal energy of $\mathrm{Ti}$ as a function of semi-lattice constant $a_{1}$. It follows from Figs. 1 and 2 that bcc lattice of Ti becomes unstable at a theoretical tensile stress of $0.3445 \times 10^{9} \mathrm{~N} / \mathrm{m}^{2}$ and a corresponding strain of $1.8 \%$ when $B B<0$ and in compression, the bcc lattice of $\mathrm{Ti}$ becomes unstable at an applied stress of $-0.5222 \times 10^{9} \mathrm{~N} / \mathrm{m}^{2}$ with a strain of $1.6 \%$ when $A A<0$ for $n=1, m=2$ and $\alpha a_{0}=1.8735$. Thus the range of stability of $\mathrm{Ti}$ is from $a_{1}=1.637376 \AA$, $a_{2}=1.676791 \AA$, to $a_{1}=1.693952 \AA, a_{2}=1.649544 \AA$.

Again it follows from Figs. 1 and 2 that the theoretical strength of bct phase of $\mathrm{Ti}$ is $1.956 \times 10^{10} \mathrm{~N} / \mathrm{m}^{2}$ in tension when $B_{23}<0$ at $a_{1}=2.3296 . \AA, a_{2}=$ $1.424506 \AA$ resulting into a peak in $B B$ at this value of $a_{1}$ and $-0.39 \times 10^{10} \mathrm{~N} / \mathrm{m}^{2}$ in compression when Eq. (4) is violated; the corresponding strains are $9.8 \%$ in tension and $-5.88 \%$ in compression. The bct phase of $\mathrm{Ti}$ is stable within the range 


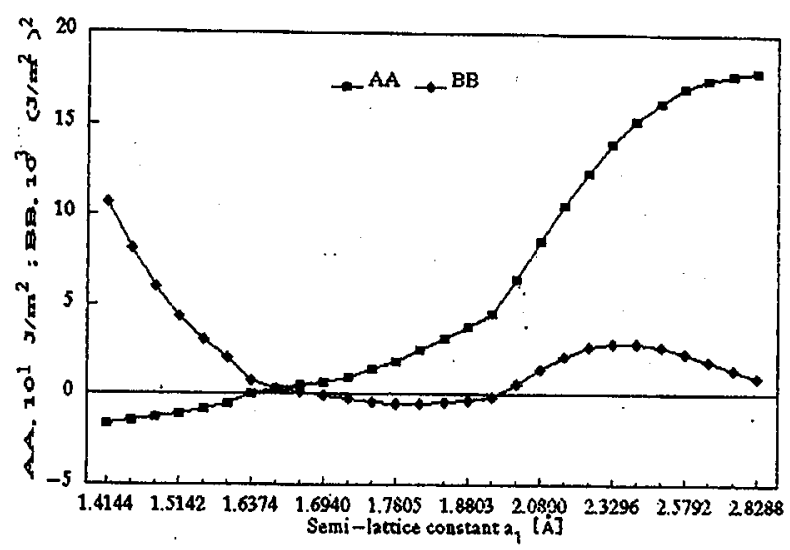

Fig. 1. Variation of $A A$ and $B B$ as a function of semi-lattice constant for Ti.

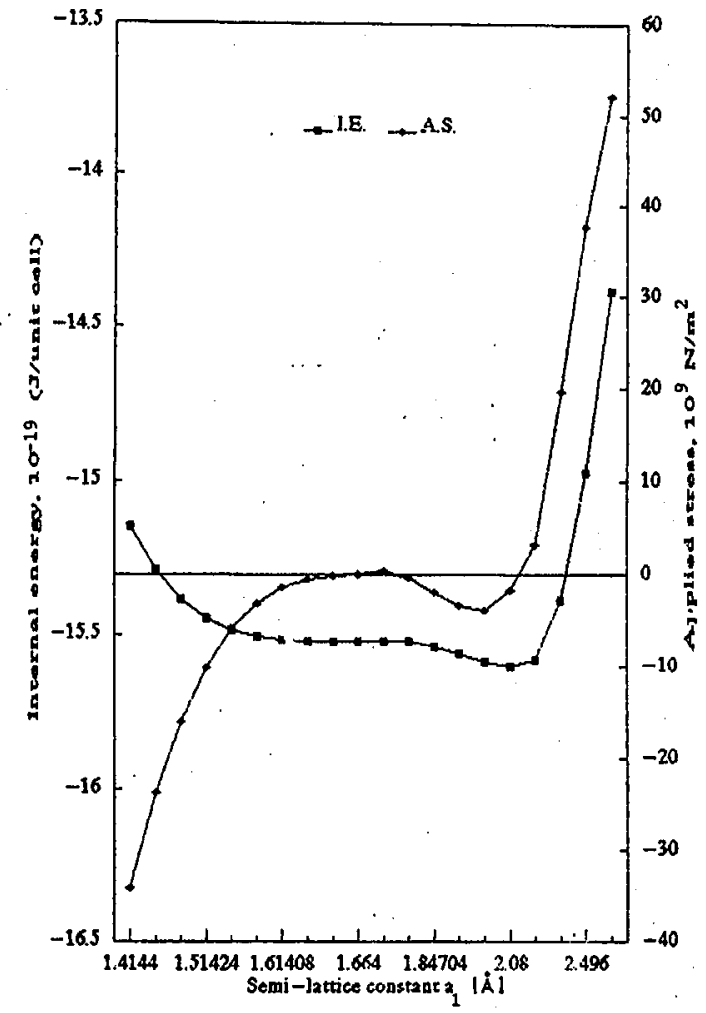

Fig. 2. Variation of internal energy and applied stress as a function of semi-lattice constant for Ti.

of semi-lattice constants $a_{1}=1.9968 \AA, a_{2}=1.512215 \AA$ to $a_{1}=2.3296 \AA$, $a_{2}=1.424506 \AA$. 


\subsection{Zirconium}

Figure 3 shows the variation of $A A=B_{22}-B_{23}$ and $B B=B_{11}\left(B_{22}+B_{23}\right)$ $-2 B_{12}^{2}$ as a function of semi-lattice constant $a_{1}$ for $\mathrm{Zr}$. Figure 4 shows the variation of applied stress and internal energy of $\mathrm{Zr}$ as a function of semi-lattice constant $a_{1}$. Figures 3 and 4 show that bcc lattice of $\mathrm{Zr}$ becomes unstable at a theoretical tensile stress of $0.9421 \times 10^{9} \mathrm{~N} / \mathrm{m}^{2}$ and a corresponding strain of $3.9 \%$ when $B B<0$ and in compression, the bcc lattice of $\mathrm{Zr}$ becomes unstable at an applied stress of $-1.9086 \times 10^{9} \mathrm{~N} / \mathrm{m}^{2}$ with a strain of $3.4 \%$ when $A A<0$ for $n=1, m=1.5$ and $\alpha a_{0}=1.7808$. Thus the range of stability of $\mathrm{Zr}$ is from $a_{1}=1.750392 \AA$, $a_{2}=1.840167 \AA$ to $a_{1}=1.882668 \AA, a_{2}=1.779211 \AA$.

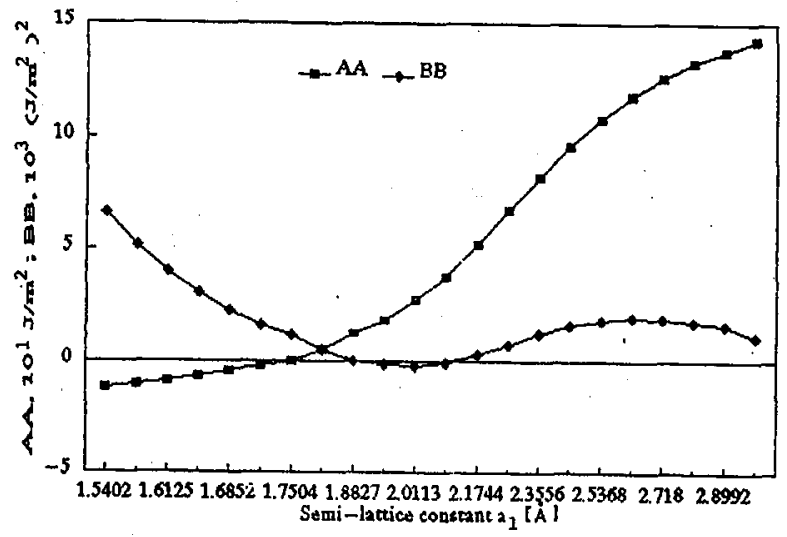

Fig. 3. Variation of $A A$ and $B B$ as a function of semi-lattice constant for Zr.

Figures 3 and 4 depict that the theoretical strength of bct phase of $\mathrm{Zr}$ is $2.074 \times 10^{10} \mathrm{~N} / \mathrm{m}^{2}$ in tension when $B_{23}<0$ at $a_{1}=2.6274 \AA, a_{2}=1.541015 \AA$ resulting into a peak in $B B$ at this value of $a_{1}$ and $-0.112 \times 10^{10} \mathrm{~N} / \mathrm{m}^{2}$ in compression when Eq. (4) is violated; the corresponding strains are $16.89 \%$ in tension and $-3.23 \%$ in compression. The bct phase of $\mathrm{Zr}$ is stable within the range of semi-lattice constants $a_{1}=2.1744 \AA, a_{2}=1.651645 \AA$ to $a_{1}=2.6274 \AA$, $a_{2}=1.541015 \AA$.

\subsection{Hafnium}

Figure 5 shows the variation of $A A=B_{22}-B_{23}$ and $B B=B_{11}\left(B_{22}+B_{23}\right)$ $-2 B_{12}^{2}$ as a function of semi-lattice constant $a_{1}$ for bcc Hf. Figure 6 shows the variation of applied stress and internal energy of bcc $\mathrm{Hf}$ as a function of semi-lattice constant $a_{1}$. It follows from Figs. 5 and 6 that bcc lattice of Hf becomes unstable at a theoretical tensile stress of $0.4078 \times 10^{9} \mathrm{~N} / \mathrm{m}^{2}$ and a corresponding strain of $1.98 \%$ when $B B<0$ and in compression, the bcc lattice of Hf becomes unstable at an applied stress of $-0.6418 \times 10^{9} \mathrm{~N} / \mathrm{m}^{2}$ with a strain of $1.82 \%$ when $A A<0$ for $n=2, m=1.5$ and $\alpha a_{0}=2.04445$. Thus the range of stability of bcc Hf is from $a_{1}=1.772149 \AA, a_{2}=1.620701 \AA$ to $a_{1}=1.840739 \AA, a_{2}=1.787827 \AA$. 


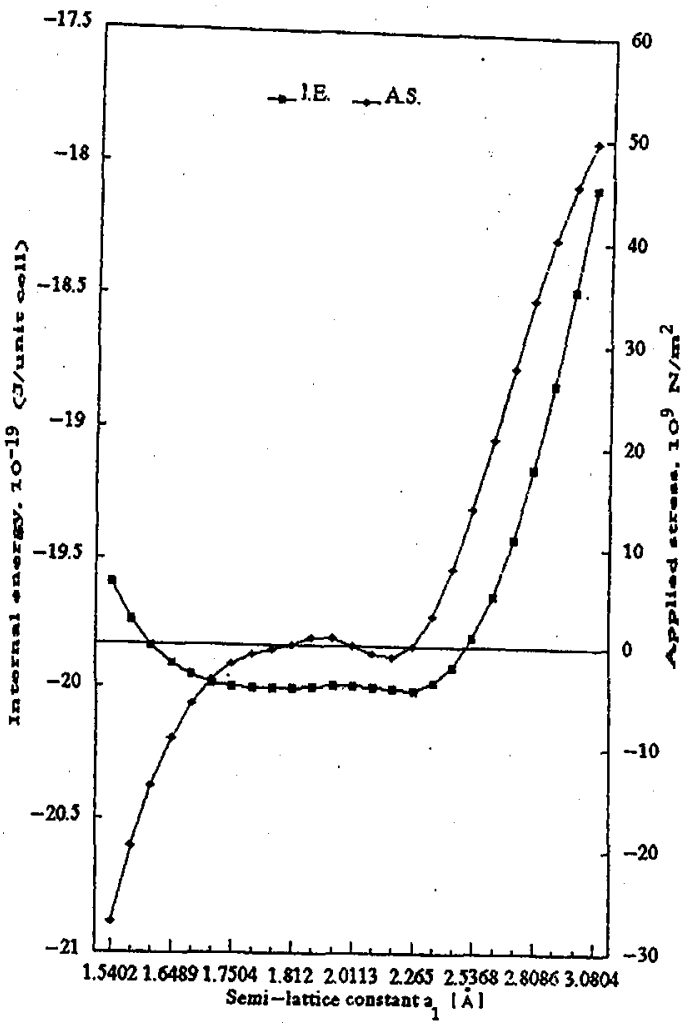

Fig. 4. Variation of internal energy and applied stress as a function of semi-lattice constant for Zr.

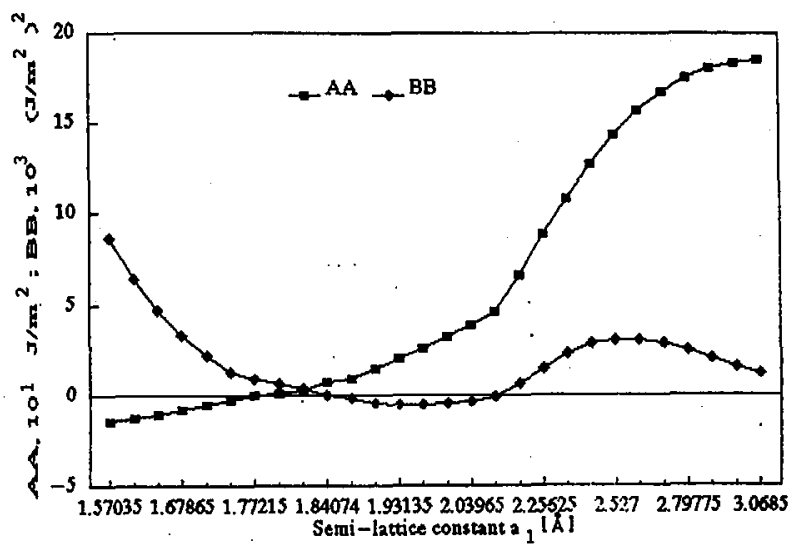

Fig. 5. Variation of $A A$ and $B B$ as a function of semi-lattice constant for Hf.

Again it follows from Figs. 5 and 6 that the theoretical strength of bct phase of Hf is $1.858 \times 10^{10} \mathrm{~N} / \mathrm{m}^{2}$ in tension when $B_{23}<0$ at $a_{1}=2.527 \AA, a_{2}=$ 


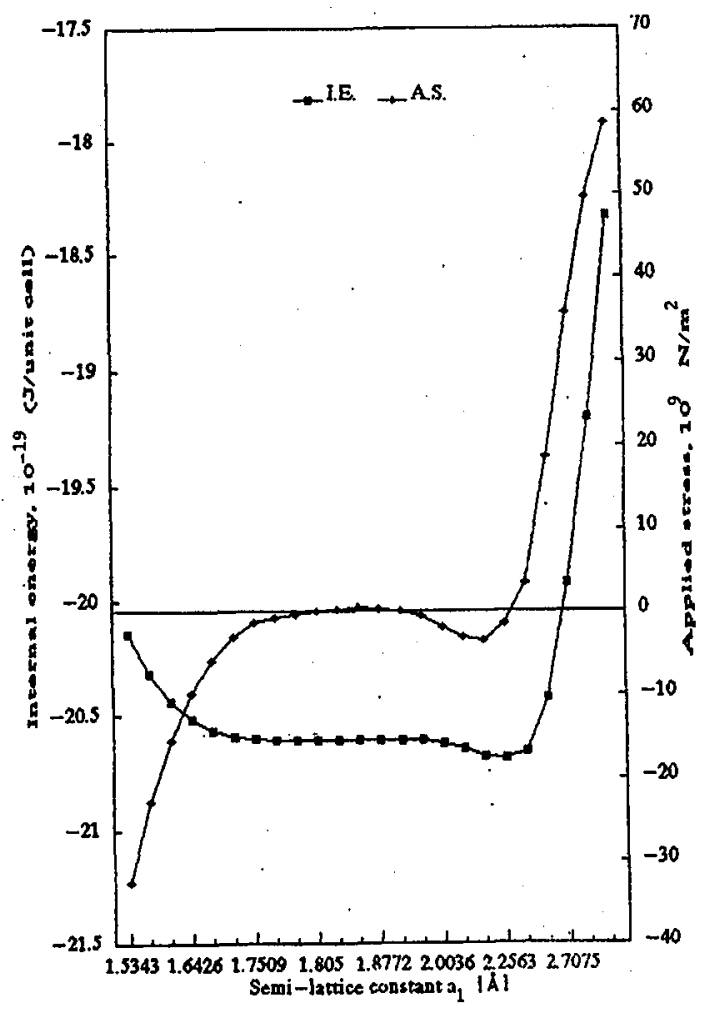

Fig. 6. Variation of internal energy and applied stress as a function of semi-lattice constant for Hf.

$1.545943 \AA$ resulting into a peak in $B B$ at this value of $a_{1}$ and $-0.349 \times 10^{10} \mathrm{~N} / \mathrm{m}^{2}$ in compression when Eq. (4) is violated; the corresponding strains are $9.8 \%$ in tension and $-5.88 \%$ in compression. The bct phase of $\mathrm{Hf}$ is stable within the range of semi-lattice constants $a_{1}=2.166 \AA, a_{2}=1.640438 \AA$ to $a_{1}=2.527 \AA$, $a_{2}=1.545943 \AA$.

\section{Conclusions}

The following main conclusions emerge on the basis of our investigations carried out for bcc metals under study of group IV:

(i) The variation of internal energy defined by Eq. (6) with semi-lattice constant $a_{1}$ for the bcc metals under study shows two distinct minima: one for bcc phase (where stress $\sigma_{1}$ becomes negative) and the other for bct phase (where stress $\sigma_{1}=0$ ). It is to be noted that the internal energy minimum (IEM) of the bct phase is considerably lower than that of the bcc phase. The unstressed $\left(\sigma_{1}=0\right)$ bct phase possesses the semi-lattice constant $a_{1}=b_{1}^{\circ}$ and $a_{2}=b_{2}^{\circ}$ corresponding to the minimum of internal energy. The values of semi-lattice constants $a_{1}, a_{2}$ and the corresponding values of the energy minima for the bcc and bct phases of the metals studied are given in Table III. 
TABLE III

Internal energy minima (IEM) and stress.

\begin{tabular}{c|c|c|c|c}
\hline \hline \multicolumn{5}{c}{ (a) bcc phase } \\
\hline Metal & $a_{1}[\AA]$ & $a_{2}[\AA]$ & $\begin{array}{c}\text { Minima position } \\
{\left[10^{-19} \mathrm{~J} / \text { unit cell }\right]}\end{array}$ & $\begin{array}{c}\text { Stress } \\
{\left[10^{9} \mathrm{~N} / \mathrm{m}^{2}\right]}\end{array}$ \\
\hline $\mathrm{Ti}$ & 1.78048 & 1.608335 & -15.522 & -0.373 \\
$\mathrm{Zr}$ & 2.04756 & 1.704053 & -19.992 & -0.336 \\
$\mathrm{Hf}$ & 1.93135 & 1.744075 & -20.608 & -0.434 \\
\hline \multicolumn{5}{|c}{ (b) bct phase } \\
\hline Metal & $a_{1}=b_{1}^{\circ}[\AA]$ & $a_{2}=b_{2}^{\circ}[\AA]$ & Minima position & Stress \\
& & & {$\left[10^{-19} \mathrm{~J} /\right.$ unit cell $]$} & {$\left[10^{9} \mathrm{~N} / \mathrm{m}^{2}\right]$} \\
\hline $\mathrm{Ti}$ & 2.121600 & 1.470275 & -15.605 & 0.0 \\
$\mathrm{Zr}$ & 2.246880 & 1.627884 & -20.007 & 0.0 \\
$\mathrm{Hf}$ & 2.301375 & 1.595221 & -20.705 & 0.0
\end{tabular}

(ii) It follows from the analysis of our results mentioned in Sec. 3 for the metals studied that the range of stability of the bct phase is considerably greater than that of the bcc phase.

\section{TABLE IV}

Computed values of $C_{11}$ and $C_{12}$ for bcc phase in

\begin{tabular}{l|l|l|l}
\multicolumn{1}{l}{$10^{11} \mathrm{~N} / \mathrm{m}^{2}}$. \\
\hline \hline Metal & \multicolumn{1}{|c|}{$C_{11}$} & \multicolumn{1}{|c}{$C_{12}$} & \multicolumn{1}{c}{ Remark } \\
\hline $\mathrm{Ti}$ & 1.2369 & 1.1484 & At equilibrium \\
& 1.1044 & 1.1453 & At failure in tension \\
& 1.3926 & 1.1499 & At failure in compression \\
Expt. & 1.34 & 1.10 & {$[25]$} \\
\hline $\mathrm{Zr}$ & 0.9613 & 0.8228 & At equilibrium \\
& 0.7535 & 0.8127 & At failure in tension \\
& 1.2610 & 0.8239 & At failure in compression \\
Expt. & 0.97 & 0.82 & [26] \\
\hline Hf & 1.1841 & 1.0904 & At equilibrium \\
& 1.0470 & 1.0855 & At failure in tension \\
& 1.3552 & 1.0912 & At failure in compression \\
Expt. & 1.31 & 1.03 & [27]
\end{tabular}

(iii) The second-order elastic constants (Table IV) $C_{11}$ and $C_{12}$ are calculated, similarly as by Milstein [18], at the stress-free equilibrium values of semi-lattice constant and at failure in tension and compression values of semi-lattice con- 
stant. It is observed that in general computed values of elastic constants $\left(C_{11}\right.$ and $\left.C_{12}\right)$ decrease in tension and increase in compression as a result of phase transitions.

\section{Acknowledgments}

Two of us (M.L.V. and A.V.) are thankful to Dr. G. Singh (Department of Physics, R.B.S. College, Agra) for many useful discussions and Mr. N.K. Khurana of Universal Computer Training Point (UCTP) for providing computation facility.

\section{References}

[1] A.A. Abrikosov, A.T. Buzdin, M.L. Kulic, D.A. Kuptsov, Int. J. Mod. Phys. B 1, 1045 (1988).

[2] Y. Syono, M. Kikuchi, K. Oh-ishi, K. Hiraga, H. Arai, Y. Matsui, N. Kobayashi, T. Sasaoka, Y. Muto, Jpn. J. Appl. Phys. (Part 2) 26, L498 (1987).

[3] F. Guinea, Int. J. Mod. Phys. B 1, 1095 (1988).

[4] D.J. Bishop, A.P. Ramirez, P.L. Gammmel, B. Batlogg, E.A. Rietman, R.J. Cava, A.J. Millis, Phys. Rev. B 36, 2408 (1987).

[5] A.G. Saif, Int J. Mod. Phys. B 1, 1121 (1988).

[6] G. Cannelli, R. Cantelli, F. Cordero, Int. J. Mod. Phys. B 1, 1157 (1988).

[7] T. Laegreid, K. Fossheim, Europhys. Lett. 6, 81 (1988).

[8] D.P. Almond, E. Lambson, G.A. Saunders, Wang Hong, J. Phys. F 17, L 221 (1987).

[9] G. Cannelli, R. Cantelli, F. Cordero, G.A. Costa, M. Ferretti, G.L. Dlcese, Europhys. Lett. 6, 271 (1988).

[10] M. Sarikaya, E. Stren, Phys. Rev. B 37, 9373 (1988).

[11] F.W. De Wette, Comments Condens. Matter Phys. (UK) 15, 225 (1991).

[12] L. Pintschovius, N. Pyka, W. Reichardt, A.Yu. Rumiantsev, N.L. Mitrofanov, A.S. Ivanov, G. Collin, P. Bourges, Physica C 185-189, 156 (1991).

[13] M. Born, R. Furth, Proc. Camb. Philos. Soc. (UK) 36, 454 (1940); A. Kelly, Strong Solids, Clarendon Press, Oxford 1966; W.R. Tyson, Philos. Mag. 14, 925 (1966); A. Kelly, W.R. Tyson, A.A. Cottrell, Philos. Mag. 15, 567 (1967); N.H. Macmillan, J. Mater. Sci. 7, 239 (1972); J. Pokluda, P. Sandera, Phys. Status Solidi B 167, 543 (1991).

[14] M. Born, Proc. Camb. Philos. Soc. (UK) 36, 160 (1940).

[15] I.H. Khlyustkov, A.T. Buzdin, Adv. Phys. 36, 271 (1987); J.G. Bednorz, K.A. Mueller, Z. Phys. B 64, 189 (1986); Z.Z. Sheng, A.M. Herman, Nature 332, 138 (1988).

[16] G.F. Taylor, Phys. Rev. 23, 655 (1924).

[17] G. Wassermann, Z. Krist. 75, 376 (1930).

[18] R.D. Misra, Proc. Camb. Philos. Soc. (UK) 36, 173 (1940); F. Milstein, Phys. Rev. B 2, 512 (1971); Phys. Rev. B 3, 1130 (1971); F. Milstein, Phys. Rev. B 10, 3635 (1974); J. Appl. Phys. 44, 3833 (1973); A.K. Mitra, P.K. Sengupta, J. Phys. F 13, 2221 (1983); G. Singh, R.P.S. Rathore, Ind. J. Pure Appl. Phys. 24, 303 (1986); K. Selay Seyoum, K.P. Thakur, D. Jha, Phys. Status Solidi B 167, 495 (1991). 
[19] G. Singh, Ind. J. Pure Appl. Phys. 28, 122 (1990); Phys. Status Solidi B 161, 145 (1990); ibid. 164, 401 (1991).

[20] A. Verma, M.L. Verma, R.P.S. Rathore, Acta Phys. Pol. A 91, 583 (1997).

[21] A. Verma, M.L. Verma, R.P.S. Rathore, Acta Phys. Pol. A 90, 547 (1996).

[22] S.S. Brenner, J. Appl. Phys. 27, 1484 (1956).

[23] S.S. Brenner, Science 128, 569 (1958).

[24] J.C. Crump, J.W. Mitchell, J. Appl. Phys. 41, 717 (1970).

[25] W. Petry, A. Heiming, J. Trampenau, M. Alba, C. Herzig, H.R. Schober, G. Vogl, Phys. Rev. B 43, 10933 (1991).

[26] A. Heiming, W. Petry, J. Trampenau, M. Alba, C. Herzig, H.R. Schober, G. Vogl, Phys. Rev. B 43, 10948 (1991).

[27] J. Trampenau, A. Heiming, M. Alba, C. Herzig, W. Miekeley, H.R. Schober, Phys. Rev. $B$ 43, 10948 (1991). 